\title{
Crystal violet reactions of coagulase negative staphylococci
}

\author{
R Freeman, D Burdess, S Smith
}

\begin{abstract}
Twenty four reference strains and 112 clinical isolates of coagulase negative staphylococci (CNS) were examined for their reactions in the crystal violet test. Some species gave a white reaction and others a purple reaction. Results were consistent and reproducible and each species gave only one pattern of crystal violet reaction. Within the limited variety of species represented in the clinical isolates, Staphylococcus saprophyticus and $S$ haemolyticus gave crystal violet purple reactions, in contrast to $S$ epidermidis, which always gave a white reaction.

Investigations suggested that the mechanism of the crystal violet test in $S$ haemolyticus may be similar to that previously described in $S$ aureus. Further work is needed to characterise the ability of crystal violet to modify $S$ epidermidis and other central nervous system species. The crystal violet reaction, which has strong associations with invasiveness, phage group susceptibilities, colonisation persistence abilities, and nosocomial origin in $S$ aureus may also be useful in studies of CNS disease.
\end{abstract}

(F Clin Pathol 1994;47:283-285)

The different appearances produced on incubation by different strains of Staphylococcus aureus, following transfer of colonial material on to nutrient agar containing $10 \mu \mathrm{g} / \mathrm{ml}$ of crystal violet, form the basis of the crystal violet reaction. Meyer suggested that purplereacting strains were of human origin ( $S$ aureus humanis), white-reacting strains were from dogs (canis) and yellow-reacting strains were of bovine origin (bovis). ${ }^{1}$ We have subsequently shown that $S$ aureus strains of undoubted human origin can produce all three crystal violet appearances and that the yellow reaction is due to good pigment production in an otherwise white-reacting strain. ${ }^{2}$ Hence we have recognised only two categories-purple and white reactors.

We have also shown that the crystal violet purple reaction is associated with hospital origin, susceptibility to phage group III phages, invasiveness, ability to colonise infants, and thereafter to persist in the colonised site. Conversely, we found that non purple-reacting strains (whether white or yellow) were significantly more likely to be of community origin, to belong to phage group II, to be asso- ciated with superficial infections and to be significantly less able to persist in sites of colonisation. The crystal violet white reaction was also significantly associated with lysis by phages 94 and $96 .^{34}$

One of the earliest reports on the behaviour of staphylococci on crystal violet-containing nutrient agar was that of Chapman and Berens in $1934,{ }^{5}$ who clearly examined coagulase negative staphylococci (CNS) as well as $S$ aureus and observed differing crystal violet reactions within the CNS group.

Because CNS are increasingly involved in serious nosocomial infections, we examined some recent isolates and some reference strains in the crystal violet reaction.

\section{Methods}

Twenty four reference strains and 112 clinical isolates of CNS were collected. The clinical isolates were obtained from blood cultures and venous catheter tips from open heart surgery patients and from peritoneal dialysis effluents from patients in the nephrology unit undergoing continuous ambulatory peritoneal dialysis (CAPD). None of the isolates was from urine specimens. All the clinical isolates had been identified by a commercial technique (API-STAPH, API Laboratories, Basingstoke, England) and were tested for slime production by a standard method. ${ }^{6}$

Organisms were stored in glycerol broth and held at $-20^{\circ} \mathrm{C}$ until required for study. Crystal violet reactions were assessed after initial recovery from the glycerol broth and a single subculture to check purity.

Crystal violet agar consisted of nutrient agar (Lab M, Bury, England) to which was added $10 \mu \mathrm{g} / \mathrm{ml}$ crystal violet (CI 2555, Sigma)-that is, a dilution of 1 part in 100000 .

Following overnight incubation at $37^{\circ} \mathrm{C}$ on nutrient agar ( $\mathrm{Lab} \mathrm{M}$ ), a visible quantity of colonial growth from individual colonies of each organism was transferred by loop and inoculated in circles of 5-6 mm diameter on to crystal violet agar. After overnight incubation at $37^{\circ} \mathrm{C}$ the resultant growth was examined and classified as purple or white (no examples of yellow reactions were seen). Several different strains were serially subcultured on nutrient agar and re-examined to confirm that individual reactions were reproducible.

Finally, two strains of $S$ epidermidis and two strains of $S$ haemolyticus were examined to determine the results of the crystal violet 
reaction under varying conditions, as previously described for $S$ aureus. ${ }^{2}$ Briefly, the crystal violet reaction was ascertained: (i) on a series of nutrient agar plates in which the crystal violet content varied from 1 in 10000 to 1 in 1000000 and (ii) on standard crystal violet medium (content of crystal violet 1 in 100000 ) with, sequentially, one, two, and three sterile cellulose acetate filters interposed between the medium and the transferred inocula. Inocula were as described for the standard crystal violet reaction.

\section{Results}

The crystal violet reaction results of all 24 reference strains and 112 clinical isolates are seen in table 1 which shows a consistent association between the crystal violet reaction and the speciation of the organisms. In the two species which were particularly well represented in the clinical collection $S$ epidermidis always gave a white reaction and $S$ haemolyticus always gave a purple reaction. The modest numbers of other species also behaved consistently and all these results agreed with the results obtained on the corresponding reference strains.

The results seen in table 2 show that varying the conditions of the crystal violet reaction, either by altering the crystal violet content of the medium or by interposing increasing numbers of cellulose acetate filters between the standard medium and the inoculum, had no effect on the crystal violet reaction of two strains of $S$ epidermidis.

In contrast, both strains of $S$ haemolyticus tested were affected by these variations in that as the crystal violet concentration was decreased or as filters were interposed, the crystal violet reaction of $S$ haemolyticus changed from purple to white.

There was no correlation between the crystal violet reactions of any of the clinical isolates and their known origin, whether this was from peritoneal dialysis effluent, blood culture, or catheter tip (data not shown).

Table 1 Crystal violet reactions of 24 reference strains and 112 clinical isolates of coagulase negative staphylococci

\begin{tabular}{|c|c|c|c|}
\hline \multirow{2}{*}{$\begin{array}{l}\text { Strain } \\
\text { identification }\end{array}$} & \multirow{2}{*}{$\begin{array}{l}\text { Number } \\
\text { tested }\end{array}$} & \multicolumn{2}{|c|}{ Crystal violet reaction } \\
\hline & & Purple & White \\
\hline $\begin{array}{l}\text { Clinical isolates } \\
S \text { capitis } \\
S \text { cohnii } \\
S \text { epidermidis } \\
S \text { haemolyticus } \\
S \text { hominis } \\
S \text { saprophyticus } \\
S \text { simulans } \\
S \text { warneri } \\
S \text { xylosus }\end{array}$ & $\begin{array}{l}2(0) \\
1(0) \\
62(54) \\
28(13) \\
6(1) \\
6(4) \\
3(0) \\
2(0) \\
2(0)\end{array}$ & $\begin{array}{l}0 \\
0 \\
0 \\
28 \\
0 \\
6 \\
0 \\
2 \\
0\end{array}$ & $\begin{array}{l}2 \\
1 \\
62 \\
0 \\
6 \\
0 \\
3 \\
0 \\
2\end{array}$ \\
\hline \multicolumn{4}{|l|}{ Reference strains } \\
\hline \multicolumn{2}{|l|}{ Purple } & \multicolumn{2}{|l|}{ White } \\
\hline $\begin{array}{l}\text { S arlettae } \\
S \text { chromogenes } \\
S \text { intermedius } \\
S \text { klooseri } \\
S \text { lugdenensis } \\
S \text { schleiferi } \\
S \text { warneri }\end{array}$ & $\begin{array}{l}\text { S auricularis } \\
S \text { gallinarum } \\
S \text { haemolyticus } \\
S \text { lentus } \\
S \text { saprophyticus } \\
S \text { sciuri }\end{array}$ & $\begin{array}{l}S \text { capitis } \\
S \text { carnosus } \\
S \text { cohnii } \\
S \text { equorum } \\
S \text { hyicus } \\
S \text { xylosus }\end{array}$ & $\begin{array}{l}S \text { caprae } \\
S \text { caseolyticus } \\
S \text { epidermidis } \\
S \text { hominis } \\
S \text { simulans }\end{array}$ \\
\hline
\end{tabular}

() indicates number of isolates for each species showing slime production.
Table 2 Effects of variations in crystal violet content and filter interposition on crystal violet reaction of $S$ epidermidis and $S$ haemolyticus

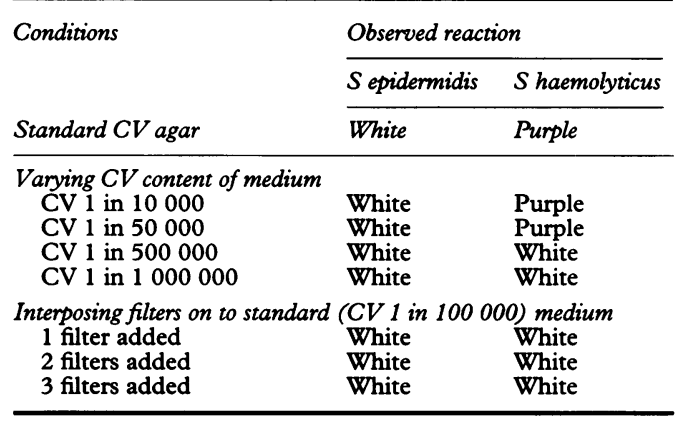

\section{Discussion}

Our results show that CNS vary in their crystal violet reaction, both purple and white reactions being obtained. No yellow reactions were seen, but unlike $S$ aureus, the standard crystal violet reaction does not apparently vary within the same species. In CNS it varies between species. Thus, for instance, all strains of $S$ epidermidis gave a white reaction whereas all strains of $S$ haemolyticus gave a purple reaction.

Our previous work on $S$ aureus showed that the crystal violet white reaction was a positive character and due to a modification of the dye, possibly by an as yet unidentified nicotinamide adenine dinucleotide (NAD) dependent enzyme system separate from the respiratory chain. ${ }^{2}$ The purple and non-purple (that is, white and yellow) biotypes of $S$ aureus represented discrete populations on a spectrum of the capacity of the species to convert crystal violet to less coloured products. Strains belonging to the purple biotype modified the crystal violet dye but only at a very slow rate. The result of the crystal violet reaction could be varied by manipulation of the inoculum or the dye content of the medium or by interposing filters between the inoculum and the crystal violet medium.

Strong associations were found in $S$ aureus between phage groups II and V and the crystal violet white-reacting phenotype, and between phage group III and the crystal violet purplereacting phenotype, but sufficient exceptions occurred to exclude an absolute dependent linkage between the phage group of individual strains of $S$ aureus and their crystal violet reaction phenotype. The picture was one of coselection of two or more independent determinants. ${ }^{2}$

In this study it was possible to alter the crystal violet purple reaction of two strains of $S$ haemolyticus in the same way as previously described for purple-reacting strains of $S$ aureus. This suggests that $S$ haemolyticus, like the purple phenotype of $S$ aureus, has the ability to modify crystal violet, but only very slowly. Representatives of other purple-reacting species, especially $S$ saprophyticus, will need to be tested to determine if this pattern is a general one.

In contrast, it proved impossible to produce conditions in which $S$ epidermidis gave a purple reaction in the crystal violet test, 
despite concentrations of crystal violet up to 1 part in 10000 being used. Under similar circumstances white-reacting strains of $S$ aureus give a purple crystal violet reaction. This suggests that the ability of $S$ epidermidis to modify crystal violet either is the same mechanism but present more abundantly than that in the white (and yellow) reacting biotype of $S$ aureus and is unable to be overwhelmed by the higher dosage of substrate, or that the crystal violet reaction of $S$ epidermidis (and possibly of the other white-reacting species of CNS) represents a different phenomenon to that of $S$ aureus and $S$ haemolyticus (and possibly of the other purple-reacting species of CNS). Further investigations along these lines may yield information of taxonomic use.

Conventionally, statements regarding the crystal violet reaction ability of strains of $S$ aureus refer to results obtained on the empirically standardised medium containing $10 \mu \mathrm{g} / \mathrm{ml}$ crystal violet. In this context our previous studies on $S$ aureus showed strong correlations within that species between crystal violet purple reactivity and strains showing evidence of invasiveness, nosocomial origin, and an ability to persist at colonised sites. ${ }^{34}$ In this study with CNS no correlation was seen between the standard crystal violet reaction and the clinical site of isolation or with the ability to produce extracellular slime. Our results show that the crystal violet reaction varies between species rather than strains, at least in those four species which were well represented in the clinical isolates ( $S$ epidermidis, $S$ haemolyticus, $S$ saprophyticus and $S$ hominis).

Previous work on CNS isolates from catheter tips from open heart surgery patients has suggested that $S$ saprophyticus (crystal violet purple) may be able to colonise and persist in such sites better than $S$ epidermidis (crystal violet white), ${ }^{7}$ and a recent survey showed that $S$ saprophyticus and $S$ haemolyticus (both crystal violet purple) were more likely to be associated with pyuria when isolated from urine. ${ }^{8} S$ haemolyticus also binds to human serum proteins much more actively than $S$ epidermidis or $S$ hominis. ${ }^{9}$ As with $S$ aureus, therefore, the picture which emerges is one suggestive of the crystal violet purple reaction being a general marker for those organisms (in this case, those species of CNS) which, each through different mechanisms, are associated with colonisation and persistence, rather than being a marker for a single virulence associated property.

Further investigations into the associations between the crystal violet reaction, species of CNS, and the disease producing roles of these organisms are necessary. It will also be important to characterise the crystal violet-modifying mechanisms of CNS and determine if they are the same in the different species of CNS and as those of $S$ aureus. This study suggests, however, that the crystal violet reaction may be an interesting tool in studies of CNS.

1 Meyer WA. A proposal for subdividing the species of $S$ aureus. Int $\mathcal{F}$ System Bacteriol 1966;17:387-401.

2 Barer MR, Burdess D, Freeman R. A study into the mechanism of the Crystal Violet reaction in Staphylococcus aureus: Epidemiol Infect 1992;109:87-96.

3 Freeman R, Hudson SJ, Burdess D. Crystal violet reactions of fresh clinical isolates of Staphylococcus aureus from two British hospitals: Epidemiol Infect 1990;105: 493-500.

4 Hudson SJ, Freeman R, Burdess D, Cookson BD. Crystal violet reactions of Staphylococcus aureus strains colonizing infants in the first six weeks. Epidemiol Infect 1993; 110:79-86.

5 Chapman GH, Berens C. Crystal violet agar as a differential medium for staphylococci: $\mathcal{f}$ Bacteriol 1934;29:437-48.

6 Christensen GD, Simpson WA, Bisno AL, Beachey EH Adherence of slime-producing strains of Staphylococcus epidermidis to smooth surfaces: Infect Immun 1982; 37:318-26. 7 Freeman $R$, Hjersing N. Species of coagulase-negative heart surgery patients: Thorax 1980;35:359-62.

8 Kleenan KT, Bannerman TL, Kloos WE. Species distribution of coagulase-negative staphylococcal isolates at a community hospital and implications for selection of staphylococcal identification procedures. $\mathcal{F}$ Clin Microbiol

9 Paulsson M, Ljungh A, Wadstrom T. Rapid identification of fibronectin, vitronectin, laminin and collagen cell surface binding proteins on coagulase-negative staphylococci by particle agglutination assays. F Clin Microbiol 1992;30:2006-12. 